\title{
Activation, Positioning or Separation Problem
}

National Cancer Institute

\section{Source}

National Cancer Institute. Activation, Positioning or Separation Problem. NCI Thesaurus.

Code 663013.

Problem associated with any deviations from the documented specifications of a medical device that relate to the sequence of events for activation, positioning or separation of device. 